\title{
Operation Efficiency Optimisation Modelling and Application of Model Predictive Control
}

\author{
Xiaohua Xia and Jiangfeng Zhang
}

\begin{abstract}
The efficiency of any energy system can be charaterised by the relevant efficiency components in terms of performance, operation, equipment and technology (POET). The overall energy efficiency of the system can be optimised by studying the POET energy efficiency components. For an existing energy system, the improvement of operation efficiency will usually be a quick win for energy efficiency. Therefore, operation efficiency improvement will be the main purpose of this paper. General procedures to establish operation efficiency optimisation models are presented. Model predictive control, a popular technique in modern control theory, is applied to solve the obtained energy models. From the case studies in water pumping systems, model predictive control will have a prosperous application in more energy efficiency problems.
\end{abstract}

Index Terms-Model predictive control (MPC), operation efficiency, energy efficiency.

\section{INTRODUCTION}

$\mathbf{W}$ ITH the increasing shortage of energy supply, energy efficiency improvement has been widely recognised as the quickest and most effective method to alleviate energy supply pressure. Energy efficiency generally consists of many components, such as management efficiency, operational efficiency, carrier efficiency, information and control efficiency, billing efficiency, maintenance efficiency, conversion efficiency, thermal efficiency, luminous efficiency, etc. In [1-3], these energy efficiency components were summarised and classified as performance efficiency, operation efficiency, equipment efficiency, and technology efficiency (POET). A prominent application of this kind of POET classification is to prevent the loss of energy efficiency improvement opportunities, which is shown in the energy audit practices ${ }^{[4]}$. This POET classification can also be applied to general energy optimisation so that all the key aspects of energy efficiency are optimised. Note the fact that proper sizing and matching of different system components, which include changing the operational schedules amongst others, for a given energy system will often save both energy and energy cost in many scenarios, therefore this paper focuses on the operation efficiency optimisation. Operation efficiency is often evaluated in terms of performance indicators such as energy, power, cost, etc. ${ }^{[1]}$. It follows that operation efficiency can usually be

This work was supported by National Research Foundation of South Africa (UID85783), the National Hub for Energy Efficiency and Demand Side Management and Exxaro.

Xiaohua Xia is with the Department of Electrical, Electronic and Computer Engineering, University of Pretoria, Pretoria 0002, South Africa (e-mail: xxia@postino.wp.ac.za).

Jiangfeng Zhang is with the Department of Electronic and Electrical Engineering, University of Strathclyde, G1 1XW, UK (e-mail: jiangfeng.zhang@strath.ac.uk). written as an optimisation problem with objective functions to be the minimisation of energy or power consumption, energy cost, etc. This kind of optimisation problem is formulated over a given time period, and can often be understood as an optimal control problem since the time dependent operation functions can be treated as the control input in optimal control. Thus various control techniques will be applicable to these energy problems. This paper focuses on the establishment of operation efficiency optimisation models and the application of model predictive control (MPC) to solve the obtained models.

MPC is well-known for its ability to use simple models, to handle constraints, and also for its closed-loop stability and inherent robustness. Therefore, MPC has become a popular tool for many industrial problems ${ }^{[5-7]}$. The MPC technique can be applied to many operation efficiency optimisation problems in which the energy systems are operated over evolving time spans. In the literature, there are various case studies on operation efficiency optimisation, and these studies include cases such as steel plant peak load management ${ }^{[8]}$, energy management of a petrochemical plant ${ }^{[9]}$, rock winder systems $^{[10]}$, water pumping systems ${ }^{[11-12]}$, power generation economic dispatch ${ }^{[13]}$, power generation maintenance ${ }^{[14]}$, etc. From these studies, it turns out that the most challenging part in the MPC applications is not the MPC itself, but the energy system modelling. Also existing studies focus on particular systems only, a general description on the operation efficiency optimisation modelling techniques is necessary. This paper summarises these modelling techniques and particularly formulates the general logic correlation constraints. These general modelling principles are illustrated by a few examples which include mineral processing, pumping systems and plant maintenance.

The paper is organized as follows. The next section provides a unified modelling framework for operation efficiency optimisation. General steps to apply MPC principles are also summarised. Section III provides some case studies, and the last section is the conclusion.

\section{OPERATION EFFICIENCY OPTIMISATION MODELLING AND MPC APPLICATIONS}

According to [1], operation efficiency is a system wide measure which is evaluated by considering the proper coordination of different system components. This coordination of system components consists of the physical, time, and human 
coordination parts. Operation efficiency has the following indicators: physical coordination indicators (sizing and matching); time coordination indicator (time control); and human coordination indicator. It is usually difficult to model the human coordinations in operation efficiency, therefore we will focus on the physical and time coordination indicators.

\section{A. Optimal Control Modelling for Operation Efficiency}

The purpose to optimise operation efficiency is usually to save energy and energy cost while at the same time to meet certain service requirement. In the following, the objective functions of the operation efficiency operation model will be chosen as both the energy and energy cost.

Assume that an energy system consists of $N$ components, each of them can be independently controlled as on or off. Whenever the $i$-th component is switched on, its power consumption will be its rated power $P_{i} \mathrm{~kW}$ for $i=1,2, \cdots, N_{1}$, and be any value between 0 and its rated power $P_{i} \mathrm{~kW}$ for $i=N_{1}+1, N_{2}+2, \cdots, N$, where $N_{1} \leq N$. The first $N_{1}$ components have only simple on/off status and include examples such as electric water heaters, electric kettles, and incandescent lights, while the last $N-N_{1}$ components have variant powers and examples can be motors controlled by variable speed drives. Let the energy price at time $t$ be $\$ p(t) /$ $\mathrm{kWh}$; then the energy consumption function $f_{E}$ and energy cost function $f_{C}$ over a fixed time interval $\left[t_{0}, t_{f}\right]$ are given below.

$$
\begin{aligned}
& f_{E}=\int_{t_{0}}^{t_{f}} \sum_{i=1}^{N} P_{i} u_{i}(t) \mathrm{d} t \\
& f_{C}=\int_{t_{0}}^{t_{f}} \sum_{i=1}^{N} P_{i} u_{i}(t) p(t) \mathrm{d} t,
\end{aligned}
$$

where $u_{i}(t)$ represents the on/off status variable and is defined as follows:

$$
u_{i}(t)\left\{\begin{array}{l}
=1, \text { if the } i \text {-th component is on and } 1 \leq i \leq N_{1} \\
=0, \text { if the } i \text {-th component is off and } 1 \leq i \leq N_{1} \\
\in[0,1], \text { if } N_{1} \leq i \leq N
\end{array}\right.
$$

The two functions $f_{E}$ and $f_{C}$ will be minimised. After formulating these two objective functions, the remaining part on the modelling is to find proper constraints. The coordination within the $N$ components of the system can be very complicated. For illustration purposes, the following typical types of coordination relations between these $N$ components are modelled.

1) Logic correlations

a) The status $u_{i}\left(t_{a}\right)$ does not affect the status of $u_{j}\left(t_{b}\right)$. For this case, we do not need to build any mathematical constraint.

b) If $u_{i}\left(t_{a}\right)$ is in the switched on status, then $u_{j}\left(t_{b}\right)$ must be in the off status. To find out a mathematical equivalent expression for this constraint, the following sign function is introduced. Let $\operatorname{sgn}(x)$ be 1 if $x>0$; 0 if $x=0$; and -1 if $x<0$. Noting the fact that $u_{i}\left(t_{a}\right)$ and $u_{j}\left(t_{b}\right)$ are nonnegative, then it follows that this constraint is equivalent to:

$$
\left(\operatorname{sgn}\left(u_{i}\left(t_{a}\right)\right)+1\right)\left(\operatorname{sgn}\left(u_{j}\left(t_{b}\right)\right)+2\right) \neq 6 .
$$

A prominent benefit to use sign function to obtain the above constraint is that this type of constraint covers the case when $i$ or $j$ is greater than $N_{1}$, that is, it covers the case where those components with variable powers are involved. An example for this type requirement can be that a piece of equipment is powered either by the grid, or by a distributed generation system, but cannot be by the two at the same time. Then the connection status of the main grid to the equipment at time $t$ corresponds to $u_{1}(t)$, while the connecting status of the distributed generation system corresponds at time $t$ to $u_{2}(t)$. This constraint following two constraints are derived as:

$$
\left(\operatorname{sgn}\left(u_{1}(t)\right)+1\right)\left(\operatorname{sgn}\left(u_{2}(t)\right)+2\right) \neq 6 \text {, for all } t .
$$

c) If $u_{i}\left(t_{a}\right)$ is in the switched on status, then $u_{j}\left(t_{b}\right)$ must be in the on status. This constraint is equivalent to the following inequality.

$$
\left(\operatorname{sgn}\left(u_{i}\left(t_{a}\right)\right)+1\right)\left(\operatorname{sgn}\left(u_{j}\left(t_{b}\right)\right)+2\right) \neq 4 .
$$

An example for this case is that at a residential home, when people switched on the TV at the lounge in the evening, they must have switched on the light in the lounge first. That is, when the status of the TV at time $t_{a}$ is on, then the status of the light must already be on at $t_{a}$.

d) If $u_{i}\left(t_{a}\right)$ is in the switched off status, then $u_{j}\left(t_{b}\right)$ must be in the on status. This constraint is equivalent to:

$$
\left(\operatorname{sgn}\left(u_{i}\left(t_{a}\right)\right)+1\right)\left(\operatorname{sgn}\left(u_{j}\left(t_{b}\right)\right)+2\right) \neq 2 .
$$

e) If $u_{i}\left(t_{a}\right)$ is in the switched off status, then $u_{j}\left(t_{b}\right)$ must be in the off status. This constraint is equivalent to:

$$
\left(\operatorname{sgn}\left(u_{i}\left(t_{a}\right)\right)+1\right)\left(\operatorname{sgn}\left(u_{j}\left(t_{b}\right)\right)+2\right) \neq 3 .
$$

2) Mass balance

Mass balance is a very common constraint in various energy systems. It can often be simplified as that at a given time period, the mass should be balanced at any system component. Mass balance equation can also be established for the overall system. For illustration purpose, we establish only the mass balance equation for a single system component:

$$
M_{i}(t+\Delta t)=M_{i}(t)+M_{i}^{\text {in }}(t)-M_{i}^{\text {out }}(t),
$$

where $M_{i}(t)$ and $M_{i}(t+\Delta t)$ are the masses of the $i$-th component at time $t$ and $t+\Delta t$, respectively; while $M_{i}^{\text {in }}(t)$ (or $M_{i}^{\text {out }}(t)$ ) is the amount of mass entered into (or left) component $i$ during the time period $(t, t+\Delta t)$. The mass $M_{i}\left(t_{0}\right)$ at the initial time $t_{0}$ is often given. The $M_{i}^{\text {in }}(t)$ and $M_{i}^{\text {out }}(t)$ are often determined by the on/off status of the $(i-1)$-th and $i$-th components, respectively. That is, there are functions $h_{i}$ and $g_{i}$ such that $M_{i}^{\text {in }}(t)=h_{i-1}\left(u_{i-1}(t)\right)$ and $M_{i}^{\text {out }}(t)=g_{i}\left(u_{i}(t)\right)$. In many applications, these $h_{i}$ and $g_{i}$ are often linear functions, and thus

$$
M_{i}^{\text {in }}(t)=a_{i-1} u_{i-1}(t), M_{i}^{\text {out }}(t)=b_{i} u_{i}(t),
$$

where $a_{i-1}$ and $b_{i}$ are constants. If there is no mass losses between the $(i-1)$-th component and the $i$-th component and ignore the time taken for the mass to flow from component $(i-1)$ into component $i$, then $M_{i-1}^{\text {out }}(t)=M_{i}^{\text {in }}(t)$. In a water pumping system, this mass balance equation is that the water volume changes in a reservoir equals the difference of the 
amount of water entering into the reservoir and the amount of water leaving the reservoir. In conveyor belt systems, the mass balance equation represents the mass changes at a stock silo equals the differences between incoming and outgoing masses to and from the stock silo.

3) Energy balance

Energy balance can be established similarly as the mass balance equation (6) either at a system component level or the overall system level. That is, the two types of energy balance equations can be briefly written as the following.

$$
\begin{aligned}
& E(t+\Delta t)=E(t)+E^{\text {in }}(t)-E^{\text {out }}(t)-E^{\text {loss }}(t), \\
& E_{i}(t+\Delta t)=E_{i}(t)+E_{i}^{\text {in }}(t)-E_{i}^{\text {out }}(t)-E_{i}^{\text {loss }}(t),
\end{aligned}
$$

where $E$ refers to energy (e.g., kinetic energy, potential energy), $E(t)$ or $E_{i}(t)$ represent the energy stored in the whole system or component $i$ at time $t$, the superscripts in, out, loss represent the energy flows into, useful energy flows out from, or energy losses at the whole system or system component during the time period $(t, t+\Delta t) . E_{i}^{\text {in }}(t)$ is usually a function of the switching status $u_{i}(t)$ and/or $E_{i-1}^{\text {out }}(t)$, i.e., there exists a function $\alpha_{i}$ such that $E_{i}^{\text {in }}(t)=\alpha_{i}\left(u_{i}(t), E_{i-1}^{\text {out }}(t)\right)$. $E_{i}^{\text {out }}(t)$ is often a function determined by the switching status $u_{i}(t)$ and/or a given external demand $D_{i}(t)$, that is, there exists a function $\beta$ such that $E_{i}^{\text {out }}(t)=\beta_{i}\left(u_{i}(t), D_{i}(t)\right)$. The energy loss $E_{i}^{\text {loss }}(t)$ is often determined by external variables such as temperature differences, humidity, pressure, material thermal convection coefficients, etc., and it is usually computable if $u_{i}(t)$ is given. Therefore, there exists a function $\gamma_{i}$ such that $E_{i}^{\text {loss }}(t)=\gamma_{i}\left(u_{i}(t)\right)$. Similarly, one can calculate $E^{\text {in }}(t), E^{\text {out }}(t)$ and $E^{\text {loss }}(t)$.

4) Process and service correlations

To meet special process or service requirements, some system components are often requested to be switched on simultaneously for a minimum time duration within a given period. This requirement is equivalent to request each of these components to be switched on for a minimum time duration at the given period. Assume that the $i$-th component must be switched for at least a duration of $\Delta T$ within the period $\left[t_{1}, t_{2}\right]$. This requirement can be formulated as the following inequality: $\int_{t_{1}}^{t_{2}} \operatorname{sgn}\left(u_{i}(t)\right) \mathrm{d} t \geq \Delta T$ There are also other types of process and service correlations, such as the delivered electrical power from a generator must meet the end user demand, an air conditioner must deliver the expected cooling load, and the pressure of compressed air must satisfy specified ranges. The corresponding constraints need to be worked out according to specific requirements.

5) Boundary constraints

There are often boundary constraints for some intermediate variables. For example, if the purpose is to save at least $10000 \mathrm{kWh}$ per year, and to save energy cost at least $\$ 10000 /$ year, then the two constraints can be written as $f_{E} \geq 10000$ and $f_{C} \geq 10000$. Other examples include the storage capacity limit of mineral silos in a conveyor belt system, reservoir capacity limit in a pumping systems, generator minimum and maximum power output, minimum and maximum temperature limits of hot water inside a water heater, steam pressure limit of a boiler, etc. These intermediate variables can usually be written as a function of the switching status $u_{i}(t), i=1, \cdots, N$, according to relevant physical dynamic processes. Generally, the following inequality is obtained:

$$
\lambda\left(u_{1}(t), u_{2}(t), \cdots, u_{N}(t)\right) \geq 0 .
$$

The above mathematical constraints provide a summary for those frequently met physical requirements in many energy systems. However, due to the complex nature of physical processes and service requirements, there will be much involved cases where none of the above derived models is directly applicable, and further analysis on the corresponding energy systems must be done.

\section{B. MPC for Optimal Control Models}

The model obtained in $(1) \sim(8)$ is an optimal control model with control variables $u_{1}(t), \cdots, u_{N}(t)$. This optimal control problem is often difficult to solve since the sign function is discontinuous and the variables $u_{1}(t), \cdots u_{N_{1}}(t)$ are binary integers. Therefore, this problem is discretised using the sampling of $t_{0}<t_{1}<\cdots<t_{m}=t_{f}, \Delta t=\left(t_{m}-t_{1}\right) / m=t_{i+1}-t_{i}$, $1 \leq i \leq m$, and then reformulated as follows:

$$
\begin{array}{ll}
\min & F_{1}(U), \\
\min & F_{2}(U), \\
\text { s.t. } & G(U) \geq 0,
\end{array}
$$

where $U=\left(u_{1}\left(t_{1}\right), u_{2}\left(t_{1}\right), \cdots, u_{N}\left(t_{1}\right), u_{1}\left(t_{2}\right), u_{2}\left(t_{2}\right)\right.$, $\left.\cdots, u_{N}\left(t_{2}\right), u_{1}\left(t_{m}\right), \cdots, u_{N}\left(t_{m}\right)\right)^{\mathrm{T}}$ is the control variable.This discrete form is easy to be implemented in MPC approach. In the MPC approach, the optimisation horizon is movable over any consecutive time length $m \Delta t=t_{m}-t_{0}$, i.e., it is solved over $\left[t_{k}, t_{m+k}\right]$ for all $k=0,1,2, \cdots$, for the corresponding optimisation problem:

$$
\begin{array}{ll}
\min & F_{1}\left(U^{k}\right), \\
\min & F_{2}\left(U^{k}\right), \\
\text { s.t. } & G\left(U^{k}\right) \geq 0,
\end{array}
$$

with the variable $U^{k}=\left(u_{1}\left(t_{k+1}\right), u_{2}\left(t_{k+1}\right), \cdots\right.$, $u_{N}\left(t_{k+1}\right), u_{1}\left(t_{k+2}\right), u_{2}\left(t_{k+2}\right), \cdots, u_{N}\left(t_{k+2}\right), u_{1}\left(t_{k+m}\right)$, $\left.\cdots, u_{N}\left(t_{k+m}\right)\right)^{\mathrm{T}}$. After obtaining the solution, $u_{1}\left(t_{k+1}\right)$, $u_{2}\left(t_{k+1}\right), \cdots, u_{N}\left(t_{k+1}\right)$ are implemented over the time period $\left[t_{k+1}, t_{k+2}\right)$. At the end of the time interval $\left[t_{k+1}, t_{k+2}\right)$, initial values are updated according to real time changes, and the above problem (10) is resolved over the time interval $\left[t_{k+1}, t_{k+m+1}\right]$ for the variable $U^{k+1}$ which is defined in the same way; see [1] for a similar MPC algorithm. The above MPC iteration steps will be applied in the case studies in Section III.

\section{CASE STUDIES}

In this section, a mineral processing system is investigated to illustrate the energy modelling procedures in Section II-A. The water purification system in [11] is restudied to propose an alternative model. The general plant maintenance problem is formulated to generalise the special case of generator maintenance in [14]. 


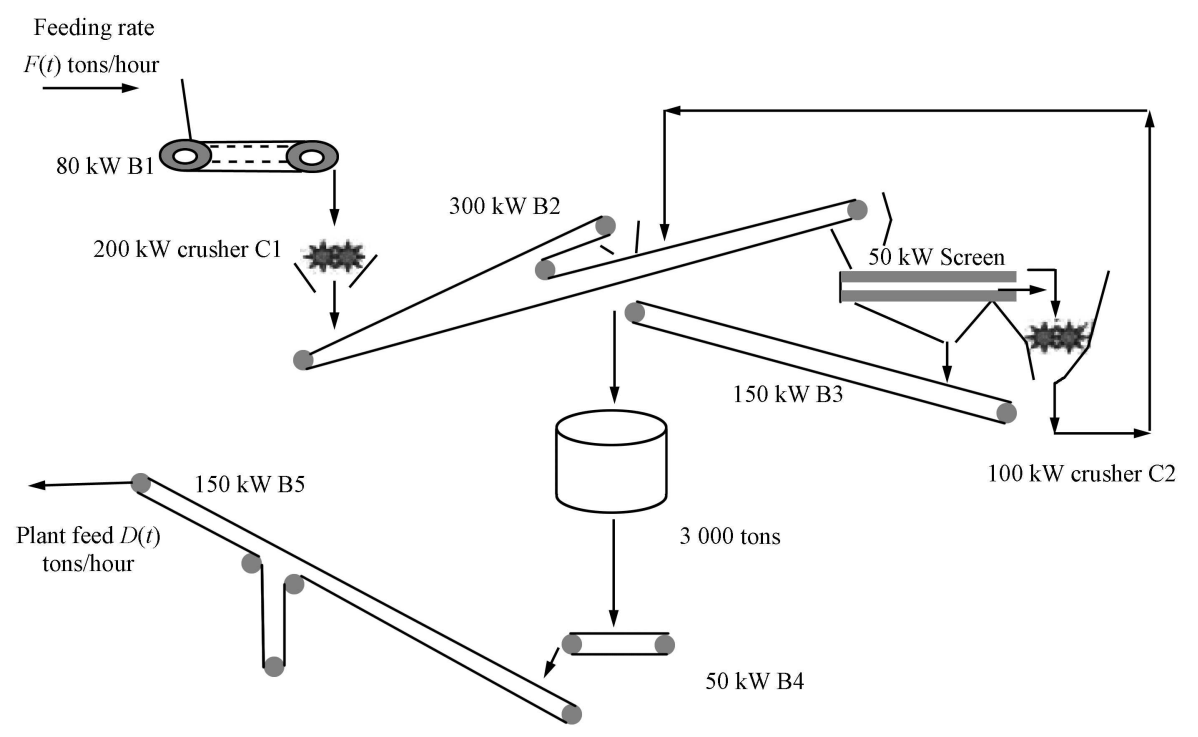

Fig. 1. Mineral processing system.

\section{A. Mineral Processing System}

In the mineral processing system in Fig. 1, minerals are fed at the rate of $F(t)$ tons/hour to the $80 \mathrm{~kW}$ conveyor belt B1. From the $200 \mathrm{~kW}$ crusher $\mathrm{C} 1$, these minerals are further transported by conveyor belt $\mathrm{B} 2$ to a $50 \mathrm{~kW}$ screen system. After the screen, smaller size minerals go to the $150 \mathrm{~kW}$ conveyor belt B3, larger size ones go to the $100 \mathrm{~kW}$ crusher $\mathrm{C} 2$ to be recrushed and then sent back to conveyor belt B2. Minerals from B3 are sent to a 3000 ton stock silo, where they will be further supplied to the $50 \mathrm{~kW}$ conveyor belt B4 and from B4 to the $150 \mathrm{~kW}$ conveyor belt $\mathrm{B} 5$. The plant feed demand at the end of conveyor belt B5 is $D(t)$ tons/hour. The question for this problem is to minimise electricity cost in terms of a time-of-use electricity tariff over the time interval $\left[t_{0}, t_{N}\right]$. Discretise $\left[t_{0}, t_{N}\right]$ as $t_{0}<t_{1}<\cdots<t_{N}, t_{1}-t_{0}=$ $t_{2}-t_{1}=\cdots=t_{N}-t_{N-1}=\Delta T$.

The overall mineral processing energy system consists of conveyors B1, B2, B3, B4, B5; crushers C1, C2; and a screen. Define the on/off switching status functions for these system components as follows. $u_{i, k}^{B}$ represents the on/off status of the $i$-th conveyor $\mathrm{B}_{i}$ at the $k$-th time interval, with $i=1,2,3,4,5$; $u_{1, k}^{C}$ and $u_{2, k}^{C}$ are the on/off status of crushers $\mathrm{C} 1$ and $\mathrm{C} 2$ at the $k$-th time interval, respectively; and $u_{k}^{S}$ is the on/off status of the screen at time $k$. The values of these switching status functions can only be 0 or 1 , representing "off" or "on" status. Following the steps in Section II-A, the objective function to minimise electricity cost over $\left[t_{0}, t_{N}\right]$ for a given electricity tariff $\$ p_{k} / \mathrm{kWh}, k=1, \cdots, N$, is written as:

$$
\begin{gathered}
\min \sum_{k=1}^{N} p_{k}\left(80 u_{1, k}^{B}+200 u_{1, k}^{C}+300 u_{2, k}^{B}+150 u_{3, k}^{B}+\right. \\
\left.100 u_{2, k}^{C}+50 u_{k}^{S}+50 u_{4, k}^{B}+150 u_{5, k}^{B}\right) \Delta t .
\end{gathered}
$$

Note in the mineral process, conveyors B1, B2, B3, crushers $\mathrm{C} 1, \mathrm{C} 2$, and the screen have the same operation schedule, i.e., they are switched on at the same time, and switched off at the same time. Similarly, conveyors B4 and B5 must also have the same operating schedule in order to minimise energy cost. Therefore, the following equalities hold:

$$
\begin{aligned}
& u_{1, k}^{B}=u_{1, k}^{C}=u_{2, k}^{B}=u_{3, k}^{B},=u_{2, k}^{C}=u_{k}^{S}, \\
& u_{4, k}^{B}=u_{5, k}^{B}, k=1, \cdots, N .
\end{aligned}
$$

From the mass balance relations, it is reasonable to assume that all minerals fed at conveyor B1 will be fed at the same rate $F(t)$ tons/hour to the 3000 ton stock silo; and similarly, the conveyor B4 must be fed at the rate of $D(t)$ tons/hour from the stock silo. Then the following mass balance relation at the stock silo can be obtained:

$$
M_{k}=M_{k-1}+F(k)-D(k), k=1, \cdots, N,
$$

where $M(k)$ represents the mass of minerals at the stock silo at time $k$, the initial mass $M(0)$ is assumed to be given. Usually the stock silo has a certain capacity constraint for safety reasons, such as the stored minerals at any time must be within the range of 10 tons to 2980 tons. Then the following constraints can be obtained.

$$
10 \leq M_{k} \leq 2980, k=1, \cdots, N .
$$

Now the optimisation problem (11) (14) can be solved to find an optimal on/off status control over the time interval $\left[t_{0}, t_{N}\right]$. An MPC algorithm can be easily designed to optimise the on/off scheduling status over the time interval $\left[t_{k}, t_{N+k}\right]$. We would however leave the MPC applications in the following two subsections.

\section{B. Water Purification System}

Fig. $\dot{2}$ is an illustration of the water pumping scheme at a water purification plant in South Africa ${ }^{[11]}$. Water flows at the rate of $40 \mathrm{~mL} /$ day (mega liter per day) from the purification plant into reservoir R1. The maximum capacity of $\mathrm{R} 1$ is $1.4 \mathrm{~mL}$, and it is also supplied with water from a fountain at the rate of $5 \mathrm{~mL} /$ day. The water from $\mathrm{R} 1$ is pumped to both reservoir R2 and reservoir R3, with the maximum capacity of $120 \mathrm{~mL}$ and $60 \mathrm{~mL}$, respectively. The water to $\mathrm{R} 2$ is pumped 
by pumps $\mathrm{K} 1, \mathrm{~K} 2$ and $\mathrm{K} 3$, each rated at $300 \mathrm{~kW}$ with the same capacity to pump $22 \mathrm{~mL} /$ day. Water from $\mathrm{R} 1$ to $\mathrm{R} 3$ is pumped by pumps G1, G2 and G3, each rated at $275 \mathrm{~kW}$ with the capacity to pump $10 \mathrm{~mL} /$ day. R2 and R3 are also supplied by a water utility called Randwater at the cost of ZAR $2.98 / \mathrm{kL}$, where ZAR represents the South African currency rand. R3 is also supplied by boreholes at a rate of $10 \mathrm{~mL} /$ day with the cost of ZAR $0.30 / \mathrm{kL}$; water cost from R1 to R2 and R3 has the same rate ZAR 1.03/kL. Pumps K3 and G3 are used as back-up pumps and usually are switched off. To simplify the model, it is assumed in [10] that pump G2 keeps running continuously, and pump K2 is chosen as the control object, and the following optimisation model is obtained.

$$
\begin{array}{ll}
\min _{u_{t}, z} & \sum_{t=1}^{T} u_{t} p c_{t}+\frac{P}{S} z C \\
\text { s.t. } & L_{1}^{t}=L_{1}^{0}+\sum_{k=1}^{t-1}\left(\mathrm{FLOWIN}_{1}^{k}-u_{k} \text { FLOWOUT }_{1}^{k}\right) \\
& 1.3 \mathrm{~mL} \geq L_{1}^{t} \geq 0.2 \mathrm{~mL}, t=1, \cdots, T \\
& \sum_{t=1+k S}^{k S+S} u_{t} P-P z_{s} \leq 0, k=0, \cdots,\left(\frac{T}{S}-1\right),
\end{array}
$$

where the two parts in the objective function represent the energy charge and the maximum demand charge, respectively, $L_{1}^{t}$ is the volume of water in reservoir R1 which should always be between $0.2 \mathrm{~mL}$ and $1.3 \mathrm{~mL}$ for capacity limit and safety reasons, $u_{t}$ is the on/off status of pump $\mathrm{K} 2, \mathrm{~S}=2$, $\mathrm{C}=\mathrm{ZAR} 66.5 / \mathrm{kW}, \mathrm{P}=300 \mathrm{~kW}$, and $z$ is an intermediate variable which helps to calculate the maximum demand. It is obvious that the constraint about water levels can be derived from the general mass balance constraint in (6). Therefore, this model is a direct application of the general principles in the previous section. Reference [11] further applies MPC to the above problem over a moving horizon of 24 hours. Percentage of savings under benchmarks with the assumptions for real value $u_{t} \in[0,1]$, the open loop control with binary values of $u_{t}$, and MPC with binary values of $u_{t}$ are compared, and Fig. 3 illustrates that MPC and open loop controller have almost the same amount of savings. However, if there is a positive random inflow disturbance, i.e., FLOWIN ${ }_{1}^{t}$ is replaced by FLOWIN $_{1}^{t}+$ $0.2 \sim$ FLOWIN $_{1}^{t} \times r(m)$ with $r(m)$ a random number between 0 and 1 , then the open loop solution will violate the reservoir allowable capacity $1.3 \mathrm{~mL}$ at $\mathrm{R} 1$ as shown in Fig. 4. Therefore, MPC method will be very helpful to provide a robust solution.

The model in (15) can be further improved by incorporating more control variables and constraints. For instance, all the four pumps G1, G2, K1, K2 can be controlled simultaneously, and the customer water demand can also be considered so as to minimise the supplementary water supply which has a high cost of R2.98/kL. Then the objective function in (15) can be revised as follows.

$$
\sum_{t=1}^{T}\left(\sum_{i=1}^{4} u_{i}^{t} p c_{t}+2.98 R_{2}^{t}+2.98 R_{3}^{t}\right)+\frac{P}{S} z C,
$$

where $u_{i}^{t}$ represents the on/off status of the four pumps at time $t, R_{2}^{t}$ and $R_{3}^{t}$ are the amount of supplementary water supplied from the water company Randwater at time $t$ to the reservoirs $\mathrm{R} 2$ and R3, respectively. Assume that customer demand at time $t$ from reservoirs $\mathrm{R} 2$ and $\mathrm{R} 3$ are $D_{2}^{t}$ and $D_{3}^{t}$, respectively. The water levels in R2 and R3 will satisfy similar constraints like those for R1 in (15). For example, the water in R2 must satisfy the following constraint.

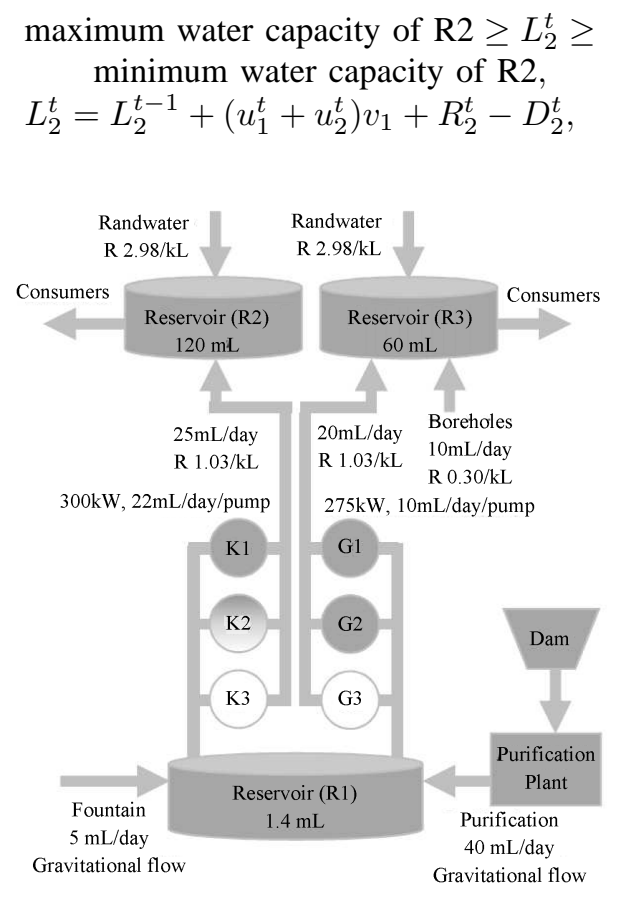

Fig. 2. A water pumping system ${ }^{[11]}$.

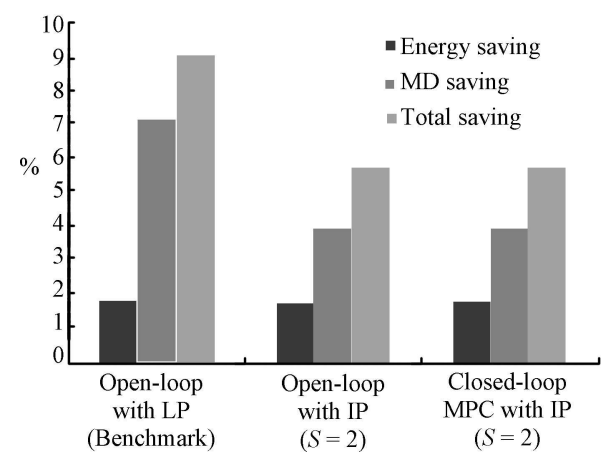

Fig. 3. Comparison of the savings by open loop controller and $\mathrm{MPC}^{[11]}$.

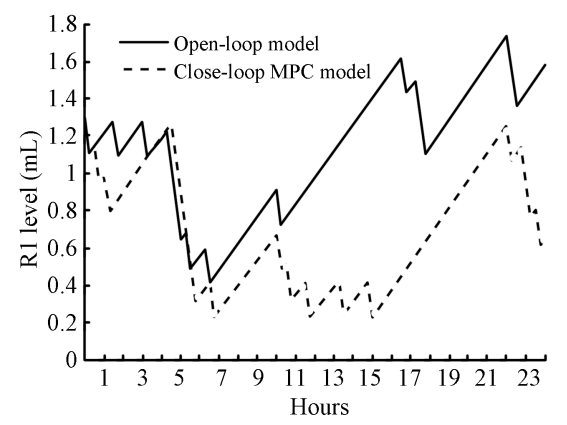

Fig. 4. Reservoir level constraint violation of open loop controllers $^{[11]}$.

where $u_{1}^{t}$ and $u_{2}^{t}$ are the on/off status of the two pumps K1 and $\mathrm{K} 2$, respectively; $v_{1}$ is the amount of water which can be 
pumped by pump K1 per unit time, which remains the same for pump K2; $D_{2}^{t}$ is the customer water demand from reservoir R2. Note that electricity suppliers often have incentives to industrial customers who are willing to reduce evening peak load, therefore it is proposed to expect the four pumps $\mathrm{K} 1$, $\mathrm{K} 2, \mathrm{G} 1, \mathrm{G} 2$ are not switched on simultaneously at the evening peak period 18:00-20:00. This can be easily formulated as the following logic correlations:

$$
u_{1}^{t}+u_{2}^{t}+u_{3}^{t}+u_{4}^{t} \leq 3 \text {, for } t \in(18: 00,20: 00) .
$$

The new model can be further applied in the MPC approach to achieve better energy savings.

\section{Plant Maintenance Optimal Scheduling}

Generator maintenance optimal scheduling has been studied by many authors; see references listed in [414]. Similar maintenance scheduling problems exist in many industrial plants. Starting from the model in [14], this subsection proposes an optimisation model to characterise the general plant maintenance scheduling problem.

Assume a plant consists of $n$ divisions (or units) which need to be regularly maintained. Consider a fixed time period of $m$ days over which an optimal maintenance schedule needs to be found. For simplicity, assume that each division needs to undergo one and only one maintenance within the $m$ days.

Let $t$ represent time (in days), and $x_{i, t}$ be the maintenance state of the $i$-th division on the $t$-th day, with $x_{i, t}=1$ representing the $i$-th division is under maintenance on the $t$-th day, while $x_{i, t}=0$ has the converse meaning. Define $y_{i, t}$ to be the start up state, with $y_{i, t}$ equal to 1 implying that the $i$-th division has been finished maintenance at time $(t-1)$ and is started to work normally at time $t$.

The objective is to minimise maintenance cost by noting the fact that each division will deliver profits at any given time, and its closing down for maintenance will cause not only the maintenance cost but also the loss of the corresponding profits. For this purpose, assume that $\$ p_{i, t}$ is the profit produced by the $i$-th division on the $t$-th day if it is operating normally. Assume that the maintenance cost for division $i$ is $\$ a_{i}$ per day, the starting up cost of division $i$ is $\$ b_{i}$. Then the objective function is formulated below.

$$
\min J=\sum_{i=1}^{n} \sum_{t=1}^{m}\left(a_{i} x_{i, t}+b_{i} y_{i, t}-p_{i, t} x_{i, t}\right) .
$$

Note that a division under maintenance cannot be started. Therefore the following constraint is obvious.

$$
x_{i, t}+y_{i, t} \leq 1,1 \leq t \leq m .
$$

Equation (21) means that the maintenance for division $i$ needs $k_{i}$ days within the $m$ days, while (22) implies that whenever the maintenance of division $i$ starts, it will take $k_{i}$ consecutive days and no interruption is allowed.

$$
\begin{gathered}
\sum_{t=1}^{m} x_{i, t}=k_{i}, 1 \leq i \leq I . \\
\sum_{t=1}^{T-k_{i}+1} x_{i, t} x_{i, t+1} \ldots x_{i, t+k_{i}-1}=1,1 \leq i \leq I .
\end{gathered}
$$

The maintenance on these divisions may be subject to certain logic correlations. For instance, the first two divisions cannot be maintained together (i.e., at least one of them must be working). This can be written as the following constraint:

$$
x_{1, t}+x_{2, t} \leq 1, \quad 1 \leq t \leq m
$$

Other types of logic correlation constraints can be formulated following the formulae in Section II-A.

The number of maintenance crew needed at any maintenance instant must not exceed the number of available crews:

$$
\begin{aligned}
& \sum_{j=1}^{n}\left(1-x_{j, t-1}\right) x_{j, t} \ldots x_{j, t+q-1} M_{j}^{q} \leq A_{t+q-1}, \\
& 1 \leq q \leq k_{i}, 2 \leq t \leq m-k_{i}+1,1 \leq i \leq n
\end{aligned}
$$

where $M_{i}^{q}$ is the number of crew needed for the $q$-th day of maintenance for the $i$-th division, and $A_{t}$ is the available number of crew at time $t$.

There might also be a least requirement on the daily profit produced even some of the divisions are under maintenance. For example, the following inequality indicates that the minimum daily profit should be at least $\$ A$.

$$
\sum_{i=1}^{n} p_{i, t}\left(1-x_{i, t}\right) \geq A, \quad 1 \leq t \leq m .
$$

Other system requirements can be added to the above model in order to determine a practically implementable scheduling plan.

The above optimisation model is formulated over the time period from $t=1$ to $t=m$, and it is easily changed into a time period starting from any day for the MPC applications. Dynamic market impact on the profit $p_{i, t}$ can be easily captured in the MPC approach, therefore, the MPC application will greatly improve the reliability of the above maintenance scheduling model.

\section{CONCLUSIONS}

This paper summarises general techniques in energy system operation efficiency modelling and the corresponding model predictive control approach to the obtained energy optimisation models. Examples from mineral processing and plant maintenance are used to illustrate the modelling process, case study on a water pumping system shows further the effectiveness of the model predictive control approach.

\section{REFERENCES}

[1] Xia X H, Zhang J F. Energy efficiency and control systems-from a POET perspective. In: Proceeding of the 2010 Conference on Control Methodologies and Technology for Energy Efficiency. Portugal: IFAC, 2010. $255-260$

[2] Xia X H, Zhang J F, Cass W. Energy management of commercial buildings - A case study from a POET perspective of energy efficiency. Journal of Energy in Southern Africa, 2012, 23(1): 23-31

[3] Xia X H, Zhang J F. Modeling and control of heavy-haul trains. IEEE Control Systems Magazine, 2011, 31(4): 18-31 
[4] Xia X H, Zhang J F. Energy auditing-from a POET perspective. In: Proceedings of the 2010 International Conference on Applied Energy. Singapore, 2010. 1200-1209

[5] Allgöwer F, Zheng A. Nonlinear Model Predictive Control. Berlin: Birkhäuser Verlag, 2000.

[6] García C E, Prett D M, Morari M. Model predictive control: theory and practice-A survey. Automatica, 1989, 25(3): 335-348

[7] Qin S J, Badgwell T A. A survey of industrial model predictive control technology. Control Engineering Practice, 2003, 11(7): 733-764

[8] Ashok S. Peak-load management in steel plants. Applied Energy, 2006, 83(5): $413-424$

[9] Wu T Y, Shieh S S, Jang S S, Liu C C L. Optimal energy management integration for a petrochemical plant under considerations of uncertain power supplies. IEEE Transactions on Power Systems, 2005, 20(3): $1431-1439$

[10] Badenhorst W, Zhang J F, Xia X H. Optimal hoist scheduling of a deep level mine twin rock winder system for demand side management. Electric Power Systems Research, 2011, 81(5): 1088-1095

[11] van Staden A J, Zhang J F, Xia X H. A model predictive control strategy for load shifting in a water pumping scheme with maximum demand charges. Applied Energy, 2011, 88(12): 4785-4794

[12] Zhang H, Xia X H, Zhang J H. Optimal sizing and operation of pumping systems to achieve energy efficiency and load shifting. Electric Power Systems Research, 2012, 86: 41-50

[13] Xia X H, Zhang J F, Elaiw A. An application of model predictive control to the dynamic economic dispatch of power generation. Control Engineering Practice, 2011, 19(6): 638-648

[14] Ekpenyong U E, Zhang JF, Xia X H. An improved robust model for generator maintenance scheduling. Electric Power Systems Research, 2012, 92: $29-36$
[15] Yamayee Z A. Maintenance scheduling: description, literature survey, and interface with overall operations scheduling. IEEE Transactions on Power Apparatus and Systems, 1982, 101(8): 2770-2779

[16] Zhang J F, Xia X H. A model predictive control approach to the periodic implementation of the solutions of the optimal dynamic resource allocation problem. Automatica, 2011, 47(2): 358-362

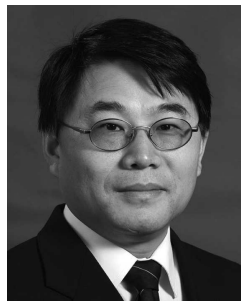

Xiaohua Xia Professor in the Department of Electrical, Electronic, and Computer Engineering at the University of Pretoria, South Africa, and the director of both the Centre of New Energy Systems and the National Hub for Energy Efficiency and Demandside Management. He was academically affiliated with the University of Stuttgart, Germany, the Ecole Centrale de Nantes, France, and the National University of Singapore before joining the University of Pretoria in 1998. He is a fellow of IEEE, a member of the Academy of Science of South Africa, and a fellow of the South African Academy of Engineering. He has been an associate editor or an editorial board member for Automatica, IEEE Transactions on Circuits and Systems II, IEEE Transactions on Automatic Control, Applied Energy, and specialist editor (control) of the SAIEE Africa Research Journal.

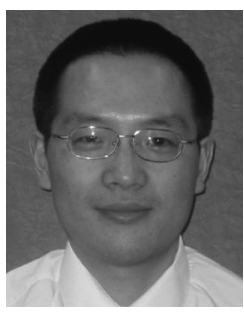

Jiangfeng Zhang obtained the B. Sc. and Ph. D. in computing mathematics from Xi'an Jiaotong University, China in July 1995 and December 1999, respectively. From February 2000 to August 2002, he was a lecturer at the Shanghai Jiaotong University. Then he was a postdoctoral researcher in the Chinese University of Hong Kong, Ecole Centrale de Nantes, Nanyang Technological University, University of Liverpool, and the University of Pretoria. He was a senior lecturer and then an associate professor in the Department of Electrical, Electronic, and Computer Engineering at the University of Pretoria from 2008 to 2013. He is now a senior lecturer at the Department of Electronic and Electrical Engineering, University of Strathclyde. His research interests include energy management and control theory. 